UNIVERSITA’ DEGLI STUDI DI ROMA “LA SAPIENZA”

DIPARTIMENTO DI STUDI GEOECONOMICI, LINGUISTICI, STATISTICI, STORICI PER L'ANALISI REGIONALE

Tel. Amm.ne 064976433 - Tel. Biblioteca 064976220 - Tel. Segr.Didattica e Studenti 0649766240 - Fax 064957606

Giovanni Di Bartolomeo

Andrew Hughes Hallett

Nicola Acocella

Working Paper $n^{\circ} 46$

Settembre 2008 


\title{
POLICY GAMES, POLICY NEUTRALITY AND \\ TINBERGEN CONTROLLABILITY UNDER \\ RATIONAL EXPECTATIONS*
}

\author{
Giovanni Di Bartolomeo \\ Andrew Hughes Hallett \\ Nicola Acocella
}

\begin{abstract}
This paper shows the relationship between static controllability (the well-known Tinbergen golden rule), and the existence and other properties of the Nash equilibrium in a dynamic setting with rational expectations for future behavior. We show how to determine the existence of equilibrium outcomes; the conditions under which no equilibrium exists; and who will get to dominate (or who will find their policies to have become ineffective) in those equilibria, without having to compute and enumerate all the possible equilibria directly.
\end{abstract}

JEL Classification: C72, E52, E61.

Keywords: Policy games, policy effectiveness, controllability, Nash equilibrium existence, rational expectations.

\footnotetext{
* Nicola Acocella and Giovanni Di Bartolomeo thank the University of Rome 'La Sapienza' for funding. Giovanni Di Bartolomeo also acknowledges the financial support of the European Union (Marie Curie ToK, contract No 014288, MTKD-CT-2004-014288) and the University of Crete for the hospitality.
} 


\title{
POLICY GAMES, POLICY NEUTRALITY AND TINBERGEN CONTROLLABILITY UNDER RATIONAL EXPECTATIONS*
}

\author{
Giovanni Di Bartolomeo ${ }^{1}$ \\ Andrew Hughes Hallett ${ }^{2}$ \\ Nicola Acocella ${ }^{3}$
}

\begin{abstract}
This paper shows the relationship between static controllability (the well-known Tinbergen golden rule), and the existence and other properties of the Nash equilibrium in a dynamic setting with rational expectations for future behavior. We show how to determine the existence of equilibrium outcomes; the conditions under which no equilibrium exists; and who will get to dominate (or who will find their policies to have become ineffective) in those equilibria, without having to compute and enumerate all the possible equilibria directly.
\end{abstract}

JEL Classification: C72, E52, E61.

Keywords: Policy games, policy effectiveness, controllability, Nash equilibrium existence, rational expectations.

\section{Introduction}

The old (classical) theory of economic policy was the joint product of a number of authors. It was developed by Tinbergen in the early 1950s when he addressed, in formal terms, the issue of the controllability of a fixed set of independent targets for a policymaker facing a parametric problem (i.e. facing an economy that could be represented by a system of linear equations describing the given conduct of other agents). A similar approach was developed by Bent Hansen in the same years (see Hansen, 1958). Tinbergen's theory however deserves the credit for having raised the problem of conditions for the existence of a first-best policy, i.e. a vector of instruments ensuring a solution to the policy problem when addressed in its simplest form with fixed targets. Theil $(1954,1956,1964)$ then gave solutions for some of the main difficulties faced by this theory. He suggested that the policymaker should maximize a preference function subject to constraints describing the functioning of the economy, and arrived at a solution of the policy problem formally very similar to that predicated by Ragnar Frisch (Frisch, 1949, 1961). ${ }^{4}$

After its Golden Age (from the 1950s to the mid 1970s), the classical theory of economic policy has been the object of fierce criticism from a number of points of view. The more general and forceful argument was raised by Lucas (1976), according to which a Tinbergen-

\footnotetext{
* Nicola Acocella and Giovanni Di Bartolomeo thank the University of Rome 'La Sapienza' for funding. Giovanni Di Bartolomeo also acknowledges the financial support of the European Union (Marie Curie ToK, contract No 014288, MTKD-CT-2004-014288) and the University of Crete for the hospitality.

${ }^{1}$ University of Teramo (e-mail: gdibartolomeo@unite.it).

${ }^{2}$ George Mason University (e-mail: ahughesh@gmu.edu).

${ }^{3}$ Sapienza University of Rome (e-mail: Nicola.acocella@unitoma1.it).

${ }^{4}$ See Hughes Hallett (1989) and Acocella and Di Bartolomeo (2007).
} 
type decision model would be inconsistent with the assumption of rational expectations. The importance of this contribution lies in the fact that it denies the validity of the solution given by Tinbergen, Theil and others, which was in the form of an (optimal) policy vector, or sequence of vectors that could achieve given policy targets (or get close) by assuming private sector behavior to be invariant to the vector itself.

After Lucas, problems of economic policy have been dealt with in a setting consistent with the criticisms raised against the classical theory. The most prominent example of this setting was the well known Barro and Gordon (1983) contribution, which is now considered to be the "father" of the policy game approach. In this approach one or many public policymakers interact among themselves and/or with other private players (e.g. firms, unions, financial markets) in a strategic way. In this context, issues of the effectiveness or neutrality of specific policy instruments have been dealt with in specific models.

Nevertheless, despite the widespread use of this game theory approach, general conditions for the (in)effectiveness of policy or equilibrium existence had never been examined. The new theory of economic policy emerging from the ashes of the classical one ${ }^{5}$, asserts general conditions for the existence of an equilibrium for the strategic interactions of the different players (i.e. the conditions which ensure that the optimal choices of each policymaker are mutually compatible), as well as the particular conditions for obtaining some specific properties associated with such interactions: for example, short-run fiscal or monetary policy neutrality or non-neutrality.

This theory can 'naturally' accommodate issues of micro-foundations of macroeconomic relations since the strategies of the different players are the outcomes of a maximizing process. In this sense it is particularly suitable for the recent development of macroeconomic literature, where the linear-quadratic approach is predominant, even if not exclusive, through the log-linearization procedure. For instance, this is the case for the New Keynesian literature and in its recent developments which analyze the interaction between monetary and fiscal authorities and labor unions (see Blanchard and Galì $(2005,2006)$ ). Further applications lie in the evolution of the literature about dynamic interactions among many monetary and/or fiscal authorities both in the new open macro-economy and in monetary union contexts; ${ }^{6}$ and in the application of robust control techniques to macroeconomics which can be modeled as a policy game between a policymaker and a fictitious evil agent. ${ }^{7}$

This paper explicitly considers the introduction of rational expectations in the new theory of economic policy. ${ }^{8}$ This step is important for at least two reasons: first, because the rational expectations were fatal for the classical theory; and second because the rational expectations assumption is the usual technique for introducing the forward looking behavior of the private sector, a vital ingredient in the recent development of modern macroeconomic theory. We want to throw light on the reasons why, and under what circumstances, we can have policy neutrality in a strategic context in an RE model. We will show that this neutrality property depends on the relationship between the number of independent instruments and the number of independent targets of the players, as in models without RE. Policy neutrality therefore depends on this aspect of the model and the associated policy problem, rather than on its dynamics or the existence of rational expectations per se.

\footnotetext{
${ }^{5}$ See Acocella and Di Bartolomeo (2006) and Acocella et al. (2006; 2007a, 2007b).

${ }^{6}$ See Pappa (2004); or Aarle et al. (2006) for a survey.

7 See, among others, Söderström (2002), Leitemo and Söderström (2004), Brock and Durlauf (2005), and Hansen and Sargent (2007).

${ }^{8}$ Indeed, the rational expectations are strictly related to the policy games since the rational expectation constraint emerges as a particular case of Stackelberg game. The agents that form rational expectations can be, in fact, considered as the game leader with respect to the action of the other agents or policymakers.
} 
This paper extends the new theory of economic policy to the case where not only do the strategic interactions between the players respect the rational expectation assumption, the constraints that they face can be derived from this assumption. We also show how our approach can be particularly useful from the point of view of model building, in order to assess some qualitative aspects of models with strategic players.

The rest of the paper is organized as follows. The next section describes the general framework of the theory of economic policy in a strategic and dynamic context. Section 3 then derives the conditions for controllability of a dynamic system with rational expectations by a single player. Section 4 considers the corresponding conditions for the existence of equilibrium outcomes and policy neutrality with multiple players. Section 5 presents some implications of our results. Section 6 shows how our theorems can be applied to a typical New Keynesian model to determine the existence (and features) of the associated policy equilibria without the need to compute an explicit solution. Section 7 concludes and hints at further generalizations and applications. The appendix describes the main argument of the new theory in formal terms, in both a static and a dynamic context.

\section{The general framework}

Before specifying our policy game framework, we need to introduce some definitions, beginning with that for controllability.

Definition (golden rule, controllability): A policymaker satisfies the golden rule of economic policy if the number of its independent instruments equals the number of its independent targets. This guarantees the controllability of the economic system, period by period.

We also define policy neutrality or ineffectiveness in a policy game context as follows. ${ }^{9}$

Definition (neutrality or ineffectiveness): A policy is neutral or ineffective if the equilibrium values of the targets are never affected by changes in the parameters of its criterion.

The classical definition of policy ineffectiveness - stating that autonomous changes in policymaker's instruments have no influence on the targets - has been modified here to fit the context of a policy game, since policy instruments in the hands of others are no longer exogenous but endogenous variables whose values depend on the preferences of other decision-makers.

Now we are ready to introduce our policy game framework. We consider a game between a set of "policymakers" that interact in a dynamic system which depends on past and expected future values of the state variables. In practice, these expectations are made by the private sector and may act as a constraint on the policy makers' choices. In this paper, there are $p$ policymakers grouped in the set $P$. Each player, $i \in P$, has a set of $m(i)$ independent policy instruments $x_{t}^{i}$; and a preference function defined on a subset of $n(i)$ linearly independent target variables, $y_{t}^{i}$, drawn from the set of all the target variables in the game: $y_{t}$. There are a total of $n$ target variables in the vector $y_{t}$.

The game is played for $T$ periods, where $T$ may be arbitrarily large. For the sake of simplicity, we will assume that $m(i) \leq n(i)$ for all players and that $m(i)$ and $n(i)$ are constant through

\footnotetext{
${ }^{9}$ See Gylfason and Lindbeck (1994).
} 
time. $^{10}$

Formally, the dynamics of the economy is represented by a linear difference equation with expectations and multiple policy makers:

$$
y_{t}=A y_{t-1}+B y_{t+1 \mid t}+\sum_{i \in P} C_{i} x_{t}^{i}+v_{t} \quad \text { for } t=1 \ldots T, \text { given } y_{0} \text { and } y_{T+1}
$$

where $y_{t+1 \mid t}=E\left[y_{t+1} \mid \Omega_{t}\right]$ denotes the mathematical expectation of $y_{t+1}$ conditional on $\Omega_{t}$ (the common information set available to all at $t$ ) and $x_{t}^{i}$ is the vector of control variables in the hands of player $i$. The matrices $A, B$ and $C_{i}$ are constant and of order $n, n$, and $n \times m(i)$, respectively, and have at least some elements which are non-zero. In this representation, $y_{0}$ is a known initial condition, and $y_{T+1}$ is an assumed or projected terminal condition. Finally, $v_{t}$ is a vector of exogenous shocks and other external influences on $y_{t}$, which have a known mean but otherwise come from an unspecified probability distribution. Note also that, if player $i$ satisfies the golden rule and has only $n(i)$ targets, $m(i)$ is equal to $n(i)$ and $C_{i}$ (after deleting the rows corresponding to the non-targets in $y_{t}$ ) is a square and invertible matrix because the instruments are linearly independent.

The preference or cost function that player $i$ aims to minimize is

$$
L^{i}\left(x^{1}, x^{2}, \ldots x^{p}\right)=E \sum_{t=1}^{T}\left(y_{t}-\bar{y}_{t}^{i}\right)^{\prime} Q_{i t}\left(y_{t}-\bar{y}_{t}^{i}\right) \quad \text { for } i \in P
$$

where $\bar{y}_{t}^{i}$ is a vector of known target values and $Q_{i t}$ is a symmetric positive semi-definite matrix of weights assigned to player $i$ 's targets in each period. For convenience we treat all the elements in $y_{t}$ to be potential targets, and $\bar{y}_{t}^{i}$ as defining player $i$ 's ideal values for them (different perhaps from the ideal values of another player), even if player $i$ subsequently chooses not to pursue $n-n(i)$ of them (corresponding to zero rows/columns in $Q_{i t}$ ) if it turns out he does not have complete controllability of $y_{t}$. Thus $Q_{i t}$ is of order $n$; but has rank $n(i) \leq$ $n$ depending on how many variables in $y_{t}$ turn out to be player $i$ 's ultimate targets (i.e. have a preference ordering defined upon them). We assume that there are at least two players which share at least one target variable. However, in order to have the potential for a conflict of interest between them, we also assume that $\bar{y}_{t}^{i} \neq \bar{y}_{t}^{j}$, for the targets held in common, for all $i \in P \neq j \in P$ in some or all periods.

Our representation is rather general. But we do not consider instrument variables in equation (2). However, to take account of the costs of using an instrument, we could simply introduce an additional target variable into equation (2), and an equality (static) constraint between it and the instrument into equation (1). That would allow us to keep instruments and targets formally separate. Note that a player facing instrument costs will not satisfy the golden rule since, in such a case, matrix $C_{i}$ will become rectangular: $\tilde{C}_{i}=\left[\left(C_{i}\right)^{\prime}: I_{m(i)}\right]^{\prime}$.

The solution of the policy game described, if it exists, will take the form of a vector of the sequences:

\footnotetext{
${ }^{10}$ We also discuss the case when the number of targets and instruments may vary during the game in section 3.2 .
} 


$$
\Pi=\left(\left\{\left(x_{t}^{1}\right)^{*}\right\}_{t=1}^{T},\left\{\left(x_{t}^{2}\right)^{*}\right\}_{i=1}^{T}, \ldots,\left\{\left(x_{t}^{p}\right)^{*}\right\}_{t=1}^{T}\right)
$$

which supports $y^{*}=\left\{y_{t}\right\}_{i=1}^{T}$, obtained by using (3) with (1) and $y_{0}$ and $y_{T+1}$.

\section{Controllability under rational expectations: The single player case}

\subsection{The model in final form}

Without loss of generality, we can write the generic linear rational expectations model, in its reduced form for a single player, as follows: ${ }^{11}$

$$
y_{t}=A y_{t-1}+B y_{t+1 \mid t}+C_{i} x_{t}^{i}+v_{t} \quad \text { for } t=1 \ldots T .
$$

Because there is only one player, we omit the index $i$ from equation (5) in this section.

This model can now be solved from the perspective of any particular period, say $t=1$, by putting it into its final form conditional on the information set available in that period: ${ }^{12}$

$$
\left(\begin{array}{c}
y_{1 \mid 1} \\
: \\
: \\
: \\
y_{T \mid 1}
\end{array}\right)=\left[\begin{array}{ccccc}
I & -B & 0 & \cdot & 0 \\
-A & I & & \cdot & \cdot \\
0 & & \cdot & & 0 \\
\cdot & \cdot & & \cdot & -B \\
0 & \cdot & 0 & -A & I
\end{array}\right]^{-1}\left\{\left[\begin{array}{ccccc}
C & 0 & \cdot & \cdot & 0 \\
0 & \cdot & & \cdot \\
\cdot & & \cdot & \cdot \\
\cdot & & & \cdot & 0 \\
0 & \cdot & \cdot & 0 & C
\end{array}\right]\left(\begin{array}{c}
x_{1 \mid 1} \\
\cdot \\
\cdot \\
\cdot \\
x_{T \mid 1}
\end{array}\right)+\left(\begin{array}{c}
v_{1 \mid 1} \\
\cdot \\
\cdot \\
\cdot \\
v_{T \mid 1}
\end{array}\right)+\left(\begin{array}{c}
A y_{0} \\
0 \\
\cdot \\
\cdot \\
0
\end{array}\right)+\left(\begin{array}{c}
0 \\
\cdot \\
\cdot \\
0 \\
B y_{T+1 \mid 1}
\end{array}\right)\right\}
$$

In this representation, $y_{0}$ is a known initial condition for $t=1$; and $y_{T+1 \mid 1}$ is an assumed or projected terminal condition - most probably one that describes the economic system's long run equilibrium state.

It is easy to show that this final form solution always exists since the inverse matrix in (5) is always well defined. To see this, define the Toeplitz matrix itself to be:

$T_{T}=\left[\begin{array}{ccccc}I & -B & 0 & \cdot & 0 \\ -A & I & & \cdot & \cdot \\ 0 & & \cdot & & 0 \\ \cdot & \cdot & & \cdot & -B \\ 0 & \cdot & 0 & -A & I\end{array}\right]$

This matrix is of order $n T$. Using the partitioning by time period, the determinant of $T_{T}$ is given by

$$
\left|T_{T-1}\right| \cdot\left|I_{n}-(-B, 0 \ldots . .0) T_{T-1}^{-1}\left(-A^{\prime}, 0 \ldots . .0\right)^{\prime}\right|
$$

However $\left|T_{T-1}\right|=\left|T_{T-2}\right|\left|I_{n}-(-B, 0 \ldots . .0) T_{T-2}^{-1}\left(-A^{\prime}, 0 \ldots . .0\right)^{\prime}\right|$, and so on. But $\left|T_{1}\right|=\left|I_{n}\right|$. Hence the inverse always exists by induction.

\footnotetext{
${ }^{11}$ The generalization of (1), and hence (5), to include any number of leads and lags is set out in the appendix.

${ }^{12}$ Hughes Hallett and Fisher (1988), Hughes Hallett et al. (1996).
} 
Given that (5) always exists, we can now write the final form model in the following way:

$$
\left(\begin{array}{c}
y_{1 \mid 1} \\
\cdot \\
\cdot \\
\cdot \\
y_{T \mid 1}
\end{array}\right)=\left[\begin{array}{ccccc}
R_{11} & \cdot & \cdot & \cdot & R_{1 T} \\
\cdot & \cdot & & & \cdot \\
\cdot & & \cdot & & \cdot \\
\cdot & & & \cdot & \cdot \\
R_{T 1} & \cdot & \cdot & \cdot & R_{T T}
\end{array}\right]\left(\begin{array}{c}
x_{1 \mid 1} \\
\cdot \\
\cdot \\
\cdot \\
x_{T \mid 1}
\end{array}\right)+\left(\begin{array}{c}
b_{1 \mid 1} \\
\cdot \\
\cdot \\
\cdot \\
b_{T \mid 1}
\end{array}\right), \quad \text { or }
$$

$$
y=R x+b
$$

where $R=T_{T}^{-1}(I \otimes C), \quad b=T_{T}^{-1}\left\{E\left(v \mid \Omega_{1}\right)+\left(A^{\prime}: 0\right)^{\prime} y_{0}+\left(0: B^{\prime}\right)^{\prime} y_{T+1 \mid 1}\right\}$, and $\otimes$ denotes the Kronecker product. In this representation of the model, each $R_{t, j}=\partial y_{t \mid 1} / \partial x_{j \mid 1}$ is an $n \times m$ matrix of policy multipliers for $t, j=1 \ldots T$. But notice that $R_{t, j} \neq 0$ even if $t<j$. Hence equation (8) implies $R_{t, j}$ is a matrix of conventional policy multipliers between $y_{t \mid 1}$ and $x_{j \mid 1}$, with a delay of $t-j$ between implementation and realization if $t \geq j$ (i.e. causality runs forwards). But $R_{t, j}$ represents a matrix of anticipatory effects, on $y_{t \mid 1}$, of an announced or anticipated policy change $x_{j \mid 1}$ at some point in the future if $t<j .{ }^{13}$

\subsection{Multi-period static controllability}

Static (or Tinbergen) controllability defines the set of conditions which must hold if an arbitrary set of target values can be achieved for the endogenous variables $y_{t}$ in each period at least in expectation given that the original model is stochastic. Define those target values to be $\bar{y}_{t \mid 1}$, where the bar denotes desired values from the perspective of period 1 , and $\bar{y}$ to be the corresponding stacked vector of desired values for all time periods.

Static controllability evidently now requires the matrix $R$ in (8) to possess an inverse:

$$
x=R^{-1}(\bar{y}-b)
$$

where $y, x$ and $b$ are all understood to be expectations conditioned on the current information set $\Omega_{t}$, as specified in (8). Hence:

Theorem 1: Under rational expectations static controllability by a single player, as in a conventional backwards looking model, requires as many independent policy instruments as there are target variables in each time period.

Proof: From (8), $R=T_{T}^{-1} C_{T}$ where $C_{T}=I_{T} \otimes C$. Hence $R_{T}^{-1}=\left(T_{T}^{-1} C_{T}\right)^{-1}=C_{T}^{-1} T_{T}$ exists if and only if $C_{T}^{-1}=I_{T} \otimes C^{-1}$ exists, since we already know that $T_{T}^{-1}$ always exists. But the instrument coefficient matrix, $C$, can only possess an inverse if $n=m$ and it has full rank: i.e. has rows and columns that are linearly independent. But those are also the conditions which provide period-by-period static controllability in a conventional backwards looking model, whether static $(A=0, B=0)$ or dynamic $(B=0, \mathrm{~A} \neq 0)$.

Corollary 1: Theorem 1 assumes that the same number of targets and instruments will be

\footnotetext{
13 As a result, a conventional "backwards looking" model will have $R_{t, j}=0$ for all $t<j$; and constant multipliers $R_{t, j}=R_{t-j}$ for $t-j=0 \ldots T-1$, if the model at (5) is linear. Neither of these things is true in (8).
} 
used in each time period since the matrix inverse of $R_{T}$ is of order $n T \times m T$. But if that is not the case, then we need $n_{t}=m_{t}$ in each period if static controllability is to hold across the whole policy interval since $I \otimes C^{-1}$ now becomes $C_{T}^{-1}=\operatorname{diag}\left\{C_{t}^{-1}\right\}$. The sufficient condition, in terms of linear independence in $C_{T}$, cannot be inferred from $R$ in this case. Similarly, if policy makers wish to pursue only $n(i)$ targets, we can delete the $n-n(i)$ rows corresponding to the non-targets in (8). Once again we need $n_{t}(i)=m_{t}$ for static controllability.

\section{Comments:}

i) Theorem 1 implies that there is no change to the static controllability conditions in a parametric context, when there are rational or forward looking expectations. As always $n=m$, well-known as the Tinbergen theorem, is a necessary condition for static controllability. The sufficient condition is linear independence in the impact of the instruments on the targets (together with $n=m$ ). This is the conventional case discussed in Hughes Hallett (1989).

ii) In the event that we have surplus instruments, $m>n$, then we may transfer $m-n$ of them from $x_{t \mid 1}$, times their coefficients from $C$, to the corresponding element of $v_{t \mid 1}$ in (6) before proceeding with theorem 1 on the reduced system.

iii) The conditions which provide multi-period static controllability in a conventional model go through unchanged in a model with rational/forward looking expectations. Theorem 1 therefore shows that there is a range of circumstances in which time inconsistency and the Lucas critique actually do not apply, despite market participants having fully forward looking expectations. Thus controllability, when it exists, allows agents to know that those actions will be undertaken since the policy makers cannot improve on them.

\section{Policy games and the golden rule: The existence of equilibria and policy neutrality} with multiple players

Given the above framework, we reintroduce the possibility of multiple policy makers whose decisions may interact. Two new theorems can be derived, which relate to the existence of the Nash equilibrium and the effectiveness of the players' policies.

Theorem 2 (non-existence): No Nash Equilibrium exists for the policy game described if at least two players having in common at least one target variable satisfy the golden rule, unless they share the same target values.

Proof. Assume that a solution (3) to the policy game exists. By focusing on player $i$, we can rewrite equation (1) as:

$$
y_{t}=A y_{t-1}+B y_{t+1 \mid t}+C_{i} x_{t}^{i}+K_{i t} \quad \text { for } t=1 \ldots T
$$

where $K_{i t}=\sum_{j \in N \mid i} C_{j} x_{t}^{i *}+v_{t}$ captures the effect of other players' decisions, and external events, on each target variable. Equation (10) can now be put into its final form conditional on the common information set available in the initial period, $\Omega_{1}$, as follows: 


$$
\left(\begin{array}{c}
y_{1 \mid 1} \\
: \\
: \\
: \\
y_{T \mid 1}
\end{array}\right)=\left[\begin{array}{ccccc}
I & -B & 0 & \cdot & 0 \\
-A & I & & \cdot & \cdot \\
0 & & \cdot & & 0 \\
\cdot & \cdot & & \cdot & -B \\
0 & \cdot & 0 & -A & I
\end{array}\right]^{-1}\left\{\left[\begin{array}{ccccc}
C_{i} & 0 & \cdot & \cdot & 0 \\
0 & \cdot & & \cdot \\
\cdot & & \cdot & & \cdot \\
\cdot & & & \cdot & 0 \\
0 & \cdot & \cdot & 0 & C_{i}
\end{array}\right]\left(\begin{array}{c}
x_{1 \mid 1}^{i} \\
\cdot \\
\cdot \\
\cdot \\
x_{T \mid 1}^{i}
\end{array}\right)+\left(\begin{array}{c}
K_{i \mid 11} \\
\cdot \\
\cdot \\
\cdot \\
K_{i T \mid 1}
\end{array}\right)+\left(\begin{array}{c}
A y_{0} \\
0 \\
\cdot \\
\cdot \\
0
\end{array}\right)+\left(\begin{array}{c}
0 \\
\cdot \\
\cdot \\
0 \\
B y_{T+1 \mid 1}
\end{array}\right)\right\}
$$

As before, this final form solution always exists since the inverse in (11) is well defined. To see that, note that the Toeplitz matrix is exactly the same as that in (6). Hence the inverse matrix in (11) always exists.

Given that the inverse of $T_{T}$ always exists, we can rewrite (11) as follows:

$$
\left(\begin{array}{c}
y_{1 \mid 1} \\
\cdot \\
\cdot \\
\cdot \\
y_{T \mid 1}
\end{array}\right)=\left[\begin{array}{ccccc}
R_{i 11} & \cdot & \cdot & \cdot & R_{i 1 T} \\
\cdot & \cdot & & \cdot \\
\cdot & & \cdot & \cdot \\
\cdot & & \cdot & \cdot \\
R_{i T 1} & \cdot & \cdot & \cdot & R_{i T T}
\end{array}\right]\left(\begin{array}{c}
x_{1 \mid 1}^{i} \\
\cdot \\
\cdot \\
\cdot \\
x_{T \mid 1}^{i}
\end{array}\right)+\left(\begin{array}{c}
b_{i \mid 1} \\
\cdot \\
\cdot \\
\cdot \\
b_{i T \mid 1}
\end{array}\right), \quad \text { or } \quad y=R_{i} x^{i}+b_{i}
$$

where $R_{i}=T_{T}^{-1} C_{i T}, b_{i}=T_{T}^{-1}\left\{E\left(K_{i} \mid \Omega_{1}\right)+\left(A^{\prime}: 0\right)^{\prime} y_{0}+\left(0: B^{\prime}\right)^{\prime} y_{T+1 \mid 1}\right\}$, and where $C_{i T}=I \otimes C_{i}$. If player $i$ satisfies the golden rule, the instrument coefficient matrix, $C_{i}$, can be inverted. If $C_{i}$ can be inverted, $C_{i T}$ can also be inverted. Hence, if player $i$ satisfies the golden rule, $R_{i}$ can be inverted too.

Theorem 2 gives a necessary condition for the existence of a policy equilibrium since it states a sufficient condition for non-existence. But it may be not sufficient for the existence of a policy equilibrium. For example, if player $j$ satisfies the golden rule, his/her optimal strategy must satisfy

$$
x^{i}=R_{i}^{-1}\left(\bar{y}^{i}-b_{i}\right) \quad \text { where } \bar{y}^{i}=\left[\bar{y}_{1}^{i^{\prime}}: \bar{y}_{2}^{i^{\prime}}: \ldots: \bar{y}_{T}^{i^{\prime}}\right]^{\prime}
$$

which implies:

$$
y=\bar{y}^{i}
$$

From equation (14) it clearly follows:

$$
y_{t \mid 1}=\bar{y}_{t}^{i} \quad \text { for } t=1 \ldots T
$$

Now assume that player $k$ also satisfies the golden rule. It would then follow that:

$$
y_{t \mid 1}=\bar{y}_{t}^{k} \quad \text { for } t=1 \ldots T
$$

However, the realized values of the target variables, at least in period 1, implied by equations (15) and (16) cannot be mutually satisfied and, therefore, no equilibrium can exist.

Hence, as a corollary from the previous theorem, we can also state:

Theorem 3 (ineffectiveness): If one (and only one) player satisfies the golden rule, all the other players' policies are ineffective with respect to the targets shared with the first player.

Proof: Obvious from theorem 2, since (15)-(16) would otherwise follow where $\bar{y}_{t}^{i} \neq \bar{y}_{t}^{k}$ in at least one element. 


\section{Comments:}

i) Theorem 3 gives a sufficient condition for policy ineffectiveness, but this does not assure the existence of an equilibrium, which may fail to occur. Theorem 2 gives a necessary condition for an equilibrium to exist because it states a sufficient condition for the opposite. Note that, if Theorem 3 is satisfied, Theorem 2 is not (and vice versa). This follows directly from the words in brackets in Theorem 3.

ii) In the Nash equilibrium, the optimal policy of each player is such as to minimize its preference function given the equilibrium policies of all the other players. In the open-loop solution, this is required in the initial period; whereas in the feedback case the condition must be satisfied in all periods.

\section{Some implications of our results}

\subsection{Equilibrium existence and the instrument costs}

It is useful to compare our results to a well-known theorem of existence of Nash equilibrium, i.e. Dasgupta and Maskin (1986), which relates the existence to the costs of the instruments since, in a similar manner, we have expressed the necessary condition for the existence in terms of an instruments/targets counting rule.

Dasgupta and Maskin (1986) in fact show that a sufficient condition for the Nash equilibrium existence is that the space of strategies of each player is convex and compact. If players' controls are unbounded, the Nash equilibrium may not exist. In static linear quadratic games, the introduction of quadratic instrument costs would make them bounded, thus assuring the existence of the equilibrium.

In our terms, the introduction of quadratic instrument costs would imply that the dimensions of matrices $C_{i}$ become $(n(i)+m(i)) \times m(i)$ as noted in section 2 . The number of instruments would then always be less than that of targets, the system would be not controllable by any player and the equilibrium would exist. From this point of view, our theorem 2 is useful, in that it displays the features of the game when it is not affected by the insertion of instrument costs. Instrument costs can alter the results and may appear to be an ad hoc assumption designed to confer different - and perhaps more appealing - features on the game. As we have it, a situation with instrument costs is just a special case. ${ }^{14}$

\subsection{Model building and the golden rule}

Theorems 2 and 3 presented above state the conditions for the consistency of the optimal strategies of all the players (and thus the existence of the equilibrium of the policy game) as well as the effectiveness of policy instruments. Their importance lies in that similar statements and conditions appear to be essential for model building both from an analytical and a policy perspective. From the former, it is interesting to know whether a specific model displays such an important feature as that of the existence of at least one equilibrium. From the latter, one should be able to understand the role of the different policymakers and their ability to have an influence (whether exclusive or joint with other players) on their target variables.

\subsection{Dynamic controllability}

\footnotetext{
${ }^{14}$ Section 2 shows this explicitly.
} 
A natural question, at this point, is to ask whether these results also extend to the concept of dynamic controllability (for which the reader is referred to Preston and Pagan, 1982, ch.8, or Holly and Hughes Hallett, 1989, ch.2). That is, do the usual conditions defining dynamic controllability also carry over to an economy subject to rational expectations and forward looking behavior? Or are they amended in some substantive way which is not apparent from a consideration of the static controllability case?

The answer is yes, they are significantly modified and in at least three different directions. First, in the presence of rational expectations, the policy authorities can use the possibility of announcements of future policy changes to help steer their policy targets towards their desired values (a possibility that has received some attention in the context of whether Central Banks should make explicit or implicit interest rate forecasts; Rudebusch and Williams, 2006). This, in effect, increases the degree of controllability and hence reduces the point at which full controllability can be achieved. But, against that, the interaction between the players can complicate the outcomes in two different ways. First, if they are sufficiently strong, those interactions may extend the period needed to achieve full controllability by one or other player, as each player struggles to offset the actions undertaken by the other in each time period. Ultimately they may destroy controllability altogether if there is no equilibrium to the game. Second, they may influence the stability of the transition path to the desired targets. If that happens, and the player with dynamic controllability finds that transition path unacceptable, he may have to modify his policies and delay full controllability (with the result that his policies will appear less effective without the other policies having become more effective).

These three new features are important, and are sufficiently complicated to require a separate paper to examine them in the required detail: see Acocella et al. (2007b). Moreover, there is an important advantage in separating dynamic from static controllability, as section 4 and the examples in section 6 make clear. Using static controllability in a multi-period dynamic setting allows us to focus on the conditions which control the existence of the eventual equilibrium and possible policy neutrality/ineffectiveness, as distinct from those which affect controllability along the way. We deal with issues of controllability in a steady state (or multiperiod certainty equivalent) sense here; and those in the conventional dynamic sense in the companion paper.

\section{Examples:}

\section{a) A simple New Keynesian model with policy interactions}

To illustrate the usefulness of our results we take a simple model with fiscal and monetary interactions, and examine the conditions under which the equilibrium may exist and cannot exist. For the sake of transparency, we work in two stages: first we analyze the case in which there are forward looking expectations but no inertia, and then the case where there are backwards looking dynamics as well. We do this to expose all the elements in the optimal policy reaction functions; these can be seen explicitly in (24) and (25) below for the first case, but the corresponding reaction functions for the second case are too complicated to give the same insight. That makes our case: it is easier to check non-existence using our targetsinstruments approach, than it is to compute the equilibrium directly. Note however that our tests rule out equilibria that do not exist (necessary conditions). Of the remainder which may exist, we can be sure that they do exist because it is easy to check directly from the model, (21) below, that the sub-matrices of $R_{T}$ are such that $R_{T}$ is then non-singular (sufficiency).

Consider a simple economy with a New Keynesian structure: 


$$
\begin{aligned}
& \pi_{t}=(1-\lambda) \beta E_{t} \pi_{t+1}+\lambda \pi_{t-1}+\kappa x_{t}+\phi f_{t}+v_{t} \\
& x_{t}=E_{t} x_{t+1}-\sigma\left(i_{t}-E_{t} \pi_{t+1}\right)+\chi f_{t}+\varepsilon_{t} \\
& \varepsilon_{t} \text { white noise. } \\
& v_{t} \text { white noise. }
\end{aligned}
$$

where $\pi_{t}$ is the inflation rate, $x_{t}$ is the output gap relative to a non-market clearing trend or a natural rate of output arising from monopolistic competition in the goods markets (Blanchard and Kiyotaki, 1987); or from tax distortions elsewhere in the economy (Alesina and Tabellini, 1987); $f_{t}$ is some kind of fiscal policy instrument; $i_{t}$ is the nominal interest rate. We assume a large value of $T$, so that the dynamic effects of any external shocks will have been absorbed before the policy exercise is complete.

This model has the following reduced form

$$
\left[\begin{array}{c}
\pi_{t} \\
x_{t}
\end{array}\right]=\left[\begin{array}{cc}
1 & -\kappa \\
0 & 1
\end{array}\right]^{-1}\left\{\left[\begin{array}{cc}
(1-\lambda) \beta & 0 \\
\sigma & 1
\end{array}\right] E_{t}\left[\begin{array}{c}
\pi_{t+1} \\
x_{t+1}
\end{array}\right]+\left[\begin{array}{cc}
\lambda & 0 \\
0 & 0
\end{array}\right]\left[\begin{array}{c}
\pi_{t-1} \\
x_{t-1}
\end{array}\right]+\left[\begin{array}{l}
\phi \\
\chi
\end{array}\right] f_{t}-\sigma\left[\begin{array}{l}
0 \\
1
\end{array}\right] i_{t}+\left[\begin{array}{c}
v_{t} \\
\varepsilon_{t}
\end{array}\right]\right\}
$$

The monetary authority's (central bank) loss is

$$
B=E_{t} \sum_{t=1}^{T} \beta^{t} \frac{1}{2}\left[\alpha_{B} x_{t}^{2}+\eta_{B} \pi_{t}^{2}\right]
$$

The fiscal authority's (government) loss function is:

$$
G=E_{t} \sum_{t=1}^{T} \beta^{t} \frac{1}{2}\left[\alpha_{G}\left(x_{t}-k\right)^{2}+\eta_{G} \pi_{t}^{2}\right] .
$$

Obviously enough, the monetary authority controls $i_{t}$, whereas $f_{t}$ is the government's control. This model is simple enough to solve for its equilibrium outcomes explicitly, when that equilibrium exists. To ensure tractability, however, we will consider the case without inertia (i.e. with purely forward looking behavior): $\lambda=0$.

In the discretionary equilibrium, the optimal fiscal policy is:

$$
\begin{aligned}
& \text { (24) } f_{t}=-\frac{\left(\alpha \chi+\kappa \eta_{G}(\kappa \chi+\phi)\right) \varepsilon_{t}}{\alpha_{G} \chi^{2}+\eta_{G}(\kappa \chi+\phi)^{2}}-\frac{\left(\alpha_{G} \chi \sigma+\eta_{G}(\kappa \chi+\phi)(\kappa \sigma+\beta)\right) E\left(\pi_{t+1}\right)}{\alpha_{G} \chi^{2}+\eta_{G}(\kappa \chi+\phi)^{2}}+ \\
& -\frac{\left(\alpha_{G} \chi+\kappa \eta_{G}(\kappa \chi+\phi)\right) E x_{t+1}}{\alpha_{G} \chi^{2}+\eta_{G}(\kappa \chi+\phi)^{2}}+\frac{\sigma\left(\alpha_{G} \chi+\kappa \eta_{G}(\kappa \chi+\phi)\right) i_{t}}{\alpha_{G} \chi^{2}+\eta_{G}(\kappa \chi+\phi)^{2}}+\frac{\alpha_{G} \chi \kappa}{\alpha_{G} \chi^{2}+\eta_{G}(\kappa \chi+\phi)^{2}}
\end{aligned}
$$

and the optimal monetary policy is:

$$
i_{t}=\frac{\varepsilon_{t}}{\sigma}+\frac{\left(\eta_{B} \kappa \beta+\alpha_{B} \sigma+\eta_{B} \kappa^{2} \sigma\right) E\left(\pi_{t+1}\right)}{\sigma\left(\alpha_{B}+\eta_{B} \kappa^{2}\right)}+\frac{\left(\alpha_{B} \chi+\eta_{B} \kappa^{2} \chi+\eta_{B} \kappa \phi\right) f_{t}}{\sigma\left(\alpha_{B}+\eta_{B} \kappa^{2}\right)}+\frac{E x_{t+1}}{\sigma}
$$

By imposing the rational expectation constraint, we can obtain the Markov Nash equilibrium results as:

$$
i_{t}=\frac{\varepsilon_{t}}{\sigma}+\frac{\alpha_{G} \chi\left(\chi \eta_{B} \kappa^{2}+\alpha_{B}(-\beta \chi+\sigma \phi+\chi)\right) k}{\left(\alpha_{B} \eta_{G}(\kappa \chi+\phi)-\alpha_{G} \chi \eta_{B} \kappa\right) \phi \sigma}
$$




$$
\begin{aligned}
& f_{t}=\frac{\left(-\beta \alpha_{B}+\alpha_{B}+\eta_{B} \kappa^{2}\right) \alpha_{G} \chi k}{\phi\left(\alpha_{B} \eta_{G}(\kappa \chi+\phi)-\alpha_{G} \chi \eta_{B} \kappa\right)} \\
& x_{t}=-\frac{\kappa \eta_{B} \alpha_{G} \chi k}{-\alpha_{G} \chi \eta_{B} \kappa+\alpha_{B} \eta_{G}(\kappa \chi+\phi)} \\
& \pi_{t}=\frac{\alpha_{B} \alpha_{G} \chi k}{-\alpha_{G} \chi \eta_{B} \kappa+\alpha_{B} \eta_{G}(\kappa \chi+\phi)}
\end{aligned}
$$

which are already fairly complicated expressions to analyze. In fact, without computing limits for particular values of the parameters, or for combinations of zero restrictions on the $\alpha$ and $\eta$ parameters, it is not immediately obvious from (26) to (29) when a policy equilibrium or equilibrium outcomes do not exist; or when one player will find that he has no influence over that part of the solution of particular concern to him. On the other hand, it would be extremely easy to ascertain the existence of policy equilibrium and/or policy neutrality by simply using our theorems. For conciseness' sake we do not perform this task here, but apply our theorems to a more complex case in which there are both rational expectations and inertia.

\section{b) A more general, but more complicated case.}

We now consider a more complex example with policy inertia:

$$
\begin{aligned}
& \text { (30) } B_{t}=E_{t} \sum_{t=1}^{T} \beta^{t} \frac{1}{2}\left[\alpha_{B}\left(x_{t}-x_{B}\right)^{2}+\eta_{B}\left(\pi_{t}-\pi_{B}\right)^{2}\right] \\
& \text { (31) } G_{t}=E_{t} \sum_{t=1}^{T} \beta^{t} \frac{1}{2}\left[\alpha_{G}\left(x_{t}-x_{G}\right)^{2}+\eta_{G}\left(\pi_{t}-\pi_{G}\right)^{2}\right] \\
& \text { (32) } \pi_{t}=(1-\lambda) \beta E_{t} \pi_{t+1}+\lambda \pi_{t-1}+\kappa x_{t}+\phi f_{t}+v_{t} \\
& \text { (33) } x_{t}=E_{t} x_{t+1}-\sigma\left(i_{t}-E_{t} \pi_{t+1}\right)+\chi f_{t}+\varepsilon_{t} \\
& \text { (34) } \varepsilon_{t} \text { realization from stochastic process of a known distribution. } \\
& \text { (35) } v_{t} \text { realization from stochastic process of a known distribution. }
\end{aligned}
$$

Note that we have two independent target variables here (inflation and output) with, by assumption, two mutually inconsistent sets of target values across the players: $x_{B} \neq x_{G}$ and $\pi_{B} \neq \pi_{G}$. And this time, there is no need to impose the additional restriction of $\lambda=0$.

As before, the model's reduced form solution demonstrates the independence of the instruments (sufficiency):

$$
\left[\begin{array}{l}
\pi_{t} \\
x_{t}
\end{array}\right]=\left[\begin{array}{cc}
1 & -\kappa \\
0 & 1
\end{array}\right]^{-1}\left\{\left[\begin{array}{cc}
1-\lambda & 0 \\
\sigma & 1
\end{array}\right] E_{t}\left[\begin{array}{c}
\pi_{t+1} \\
x_{t+1}
\end{array}\right]+\left[\begin{array}{cc}
\lambda & 0 \\
0 & 0
\end{array}\right]\left[\begin{array}{c}
\pi_{t-1} \\
x_{t-1}
\end{array}\right]+\left[\begin{array}{l}
\phi \\
\chi
\end{array}\right] f_{t}-\sigma\left[\begin{array}{l}
0 \\
1
\end{array}\right] i_{t}+\left[\begin{array}{c}
v_{t} \\
\varepsilon_{t}
\end{array}\right]\right\}
$$

Thus, and without the need to compute an explicit solution, the model's policy equilibria can be summarized as follows by using our theorems and target-instrument counting rules.

\section{Table 1 - A complete taxonomy}

\section{Game:}


1. $a_{B}, h_{B}, a_{G}, h_{G} \neq 0 \quad$ Both authorities affect output Both authorities affect inflation

2. $a_{B}=0 \quad$ Both authorities affect output $\quad p=p_{B}$

3. $h_{B}=0 \quad x=x_{B} \quad$ Both authorities affect inflation

4. $a_{G}=0 \quad$ Both authorities affect output $\quad p=p_{G}$

5. $h_{G}=0 \quad x=x_{G} \quad$ Both authorities affect inflation

6. $a_{B}=0$ and $a_{G}=0 \quad$ The equilibrium does not exist

7. $h_{B}=0$ and $h_{G}=0 \quad$ The equilibrium does not exist

8. $a_{B}=0$ and $h_{G}=0 \quad x=x_{G} \quad p=p_{B}$

9. $h_{B}=0$ and $a_{G}=0 \quad x=x_{B} \quad p=p_{G}$

Hence the value of our results is that they allow us to determine a) when an equilibrium does not exist; and b) who will find that their policies are ineffective, if it exists.

In support of table 1, it is important to note that some of the cases identified are unlikely to be encountered in practice. But many of them are cases that we deal with in the literature every day - including those where an equilibrium does not exist, or where one player finds his policies to be ineffective and therefore subject to time inconsistency by others. Case 1, "no overall control", is evidently the most general specification. But the literature on independent and conservative central banks fits into case 2: the central bank targets low inflation exclusively. Cases 3 and 4, with the reverse assignments, appear unlikely.

Cases 6 and 7, where the equilibrium outcomes do not exist, are perhaps the most interesting. Case 6 is where both policy authorities agree that inflation is the overriding problem, albeit with differing target values or priorities (Europe in the 1980s; US in the 1970s, South America post-1950; Germany in the 1920s). Likewise, case 7 is the case where both agree that recession/depression is the main problem (Japan in the 1990s, the United States and Europe in the 1930s and in 2001-3). The last two examples then define policy assignments that "decouple" the policy problem into two separate components. Case 8 has monetary policy assigned to inflation control, and fiscal policy to output stabilization - a textbook paradigm often used by policy makers. For example, the Euro-area is predicated on governments being concerned only with output stabilization since, by statute, they can have no influence on the common monetary policy ${ }^{15}$, while the ECB is designed to focus on inflation control lexicographically over output stabilization. Finally, case 9 has the reverse assignment (sometimes discussed in the context of exchange rate targeting).

\section{c) The role of instrument costs: a further extension}

Adding instrument costs produces the following economic model

$$
B_{t}=E_{t} \sum_{t=1}^{T} \beta^{t} \frac{1}{2}\left[\alpha_{B}\left(x_{t}-x_{B}\right)^{2}+\eta_{B}\left(\pi_{t}-\pi_{B}\right)^{2}+\theta_{B} s_{t}^{2}\right]
$$

\footnotetext{
${ }^{15}$ However, being unable to influence inflation directly (they have no instrument assigned to that target) does not mean that their actions do not affect inflation or that they do not account for inflation when setting fiscal policy. They do affect inflation, and take that into account, because of the spillovers from the actions induced in their opponent: see the second term in (24).
} 


$$
\begin{aligned}
G_{t} & =E_{t} \sum_{t=1}^{T} \beta^{t} \frac{1}{2}\left[\alpha_{G}\left(x_{t}-x_{G}\right)^{2}+\eta_{G}\left(\pi_{t}-\pi_{G}\right)^{2}+\theta_{G} b_{t}^{2}\right] \\
\pi_{t} & =(1-\lambda) \beta E_{t} \pi_{t+1}+\lambda \pi_{t-1}+\kappa x_{t}+\phi f_{t}+v_{t} \\
x_{t} & =E_{t} x_{t}-\sigma\left(i_{t}-E_{t} \pi_{t+1}\right)+\chi f_{t}+\varepsilon_{t} \\
S_{t} & =i_{t} \\
b_{t} & =f_{t}
\end{aligned}
$$

Now the equilibrium always exists, because no player can control the system, unless we have the two cases in which the policy problem separates into two parts: $\left(a_{G}=0, h_{B}=0\right)$ and $\left(a_{B}=0, h_{G}=0\right)$. But in these two cases, where both players care only about their own controls and both can control their own subsystems, the equilibrium will again exist since there are no shared target variables between the players. But it is not one in which the players can get exactly what they want.

\section{Concluding remarks}

This paper generalizes the results of the new theory of economic policy to the case of policy games in a dynamic setting with rational expectations for future behavior.

The importance of the new theory of economic policy lies first in the fact that it settles an old issue (policy controllability) in the only setting (policy games) in which it can be consistently placed to overcome the Lucas critique. In addition, it does so by returning to the propositions of the classical theory, which is of interest from the point of view of the history of economic thought. Third and more importantly, its two fundamental propositions appear to be essential for model building, since they state the conditions for the consistency of the optimal strategies of all the players (and thus the existence of the equilibrium of the game) as well as for the effectiveness of policy instruments.

Our paper has analyzed the problems that can arise in a policy game with a dynamic setting and rational expectations about future behavior. We have stated the relationship between the static controllability (the well-known Tinbergen golden rule) and the existence of a Nash equilibrium, as well as the particular conditions for obtaining some specific properties associated with the strategic interactions: for example, short-run fiscal or monetary policy neutrality or non-neutrality. With reference to a standard New Keynesian model, we have shown how to determine the existence of equilibrium outcomes; the conditions under which no equilibrium exists; and who gets to dominate (or equivalently, who will find their policies to have become ineffective) in those equilibria, without having to compute all the possible equilibria directly.

\section{References}

Aarle, B. van, G. Di Bartolomeo, J. Engwerda, T. Michalak, J. Plasmans (2006), Dynamic modeling of monetary and fiscal cooperation among nations, Berlin: Springer.

Acocella, N. and G. Di Bartolomeo (2006), "Tinbergen and Theil meet Nash: Controllability in policy games," Economics Letters, 90: 213-218. 
Acocella, N., G. Di Bartolomeo (2007), "Towards a new theory of economic policy: continuity and innovation," in R. Neck, C. Richter and Peter Mooslechner, eds., Quantitative economic policy Theory and applications. Essays in honour of Andrew Hughes Hallett, Berlin: Springer, 15-34.

Acocella, N., G. Di Bartolomeo and A. Hughes Hallett (2006), "Controllability in policy games: Policy neutrality and the theory of economic policy revisited," Computational Economics, 28: 91-112.

Acocella, N., G. Di Bartolomeo and A. Hughes Hallett (2007a), "Dynamic controllability with overlapping targets: Or why target independence may not be good for you," Macroeconomic Dynamics, 11: 202-213.

Acocella, N., G. Di Bartolomeo and A. Hughes Hallett (2007b), "Dynamic (target) controllability in a strategic environment," mimeo.

Alesina, A. and G. Tabellini (1987), "Rules and discretion with noncoordinated monetary and fiscal policies," Economic Inquiry, 25: 619-630.

Barro, R.J. and D. Gordon (1983), "Rules, discretion and reputation in a model of monetary policy,” Journal of Monetary Economics, 12: 101-120.

Blanchard, O.J. and J. Galì (2005), "Real wage rigidities and the New Keynesian model," MIT Department of Economics Working Paper No. 05-28; FRB Boston Working Paper No. 05-14, forthcoming in conference volume, Quantitative evidence on price determination, Journal of Money, Credit and Banking.

Blanchard, O.J. and J. Galì (2006), "A New Keynesian model with unemployment," MIT Department of Economics Working Paper No. 05-28; FRB Boston Working Paper No. 06-22.

Blanchard, O.J. and N. Kiyotaki (1987), "Monopolistic competition and the effects of aggregate demand," American Economic Review, 77: 647-666.

Brock W.A. and S.N. Durlauf (2005), "Local robustness analysis: Theory and applications," Journal of Economic and Dynamic Control, 29: 2067-2092.

Dasgupta, P. and E. Maskin, (1986), "The existence of the equilibrium in discontinuous economic games, I: Theory," Review of Economic Studies, 53: 1-26.

Frisch, R. (1949), "A memorandum on price-wage-tax subsidy policies as instruments in maintaining optimal employment," UN Document E (CN1/Dub 2), New York, reprinted as Memorandum from Universitets Socialokonomiske Institutt, Oslo, 1953.

Frisch, R. (1961), "A survey of types of economic forecasting and programming and a brief discussion of the Oslo channel model," Memorandum from the Institute of Economics at the University of Oslo, 13 May.

Gylfason, G. and A. Lindbeck (1994), "The interaction of monetary policy and wages," Public Choice, 79: 33-46.

Hansen, B. (1958), The economic theory of fiscal policy, London: Allen \& Unwin.

Hansen, L.P. and T.J. Sargent (2007), Robustness, Princeton: Princeton University Press.

Holly, S. and A. Hughes Hallett (1989), Optimal control, expectations and uncertainty, Cambridge: Cambridge University Press.

Hughes Hallett, A.J (1989), "Econometrics and the theory of economic policy: the TinbergenTheil contributions 40 years on," Oxford Economic Papers, 41: 189-214. 
Hughes Hallett, A.J and P. G. Fisher (1988), "Efficient solution techniques for linear and nonlinear rational expectations models," Journal of Economic Dynamics and Control, 12: 635657.

Hughes Hallett, A.J., Y Ma and Y. P. Yin (1996), "Hybrid algorithms with automatic switching for solving nonlinear equation systems in economics," Journal of Economic Dynamics and Control, 20: 1051-71.

Leitemo, K. and U. Söderström (2004), "Robust monetary policy in the New-Keynesian framework," CEPR Discussion Paper No. 4805, forthcoming, Macroeconomic Dynamics.

Lucas, R.E. (1976), "Econometric policy evaluation. A critique," Journal of Monetary Economics, Supplement, Carnegie-Rochester Conference Series on Public Policy, 1: 19-46.

Pappa, E. (2004), "Do the ECB and the Fed really need to cooperate? Optimal monetary policy in a two-country world," Journal of Monetary Economics, 51: 753-779.

Preston, A.J. and A.R. Pagan (1982), The theory of economic policy, Cambridge: Cambridge University Press.

Rudebusch, G. and J. Williams (2006), "Revealing secrets of the temple: The value of publishing central bank interest rate projections," NBER Working Paper 12638, Cambridge, MA.

Söderström, U. (2002), "Monetary policy with uncertain parameters," Scandinavian Journal of Economics, 104: 125-145.

Theil, H. (1954), "Econometric models and welfare maximization," Weltwirtschaftliches Archiv, 72: 60-83.

Theil, H. (1956), "On the theory of economic policy," American Economic Review, 46: 360366.

Theil, H. (1964), Optimal decision rules for government and industry, Amsterdam: North Holland. 


\section{Appendix: A generalization to multiple leads and lags}

We now consider a general linear rational expectations model, with $\mathrm{p}$ lags and $\mathrm{q}$ lead or expectations terms. This can be converted to a first order model, such as in (1), as follows. The $(p, q)$ model is of the form:
(A1) $y_{t}=A(L) y_{t}+B\left(L^{-1}\right) y_{t / t}+\sum_{i \in P} C_{i} x_{t}^{i}+v_{t}$
$t=1 \ldots T$

where $A(L)=A_{0}+A_{1} L+A_{2} L^{2}+\ldots .+A_{p} L^{p} \quad$ with $A_{0}=0$;

and $\quad B\left(L^{-1}\right)=B_{0}+B_{1} L^{-1}+\ldots \ldots . .+B_{q} L^{-q} \quad$ with $B_{0}=0$

are both polynomials in the lag operator $L y_{t}=y_{t-1}$. In such a model, we can always rewrite (A1) by stacking the variables as follows:

(A2)

$$
\left(\begin{array}{c}
y_{t+q-1} \\
\cdot \\
y_{t} \\
\cdot \\
y_{t-p+1}
\end{array}\right)=\left[\begin{array}{ccccc}
0 & \cdot & \cdot & \cdot & 0 \\
\cdot & 0 & \cdot & \cdot & 0 \\
\cdot & A_{1} & \cdot & \cdot & A_{p} \\
\cdot & I & 0 & \cdot & 0 \\
0 & \cdot & 0 & I & 0
\end{array}\right]\left(\begin{array}{c}
y_{t+q-2} \\
\cdot \\
y_{t-1} \\
\cdot \\
y_{t-p}
\end{array}\right)+\left[\begin{array}{ccccc}
0 & I & 0 & \cdot & 0 \\
\cdot & \cdot & 0 & I & 0 \\
B_{q} & \cdot & \cdot & B_{1} & 0 \\
0 & \cdot & \cdot & \cdot & 0 \\
0 & \cdot & \cdot & \cdot & 0
\end{array}\right]\left(\begin{array}{c}
y_{t+q / t} \\
\cdot \\
y_{t+1 / t} \\
\cdot \\
y_{t-p+2}
\end{array}\right)+\sum_{i \in P} C_{i} x_{t}^{i}+v_{t} .
$$

Or, in obvious notation,

$$
\text { (A3) } \tilde{y}_{t}=\tilde{A} \tilde{y}_{t-1}+\tilde{B} y_{t+1 / t}+\sum_{i \in P} C_{i} x_{t}^{i}+v_{t}
$$

which is in exactly the same form as the model at (1) of the main text. 